\title{
The Physiology of the Kidney
}

Occasionally, an issue of Physiology will focus on a subdiscipline of physiology. This issue, which broadly focuses on the kidney, is such an example.

The first of the two Emerging Topics-by Komlosi, Fintha, and Bell-addresses the mechanism of tubuloglomerular feedback (TGF). TGF is an intrinsic renal mechanism that stabilizes glomerular filtration rate (GFR) at the single-nephron level. An increase in GFR will increase the flow of tubule fluid past the macula densa cells at the end of the thick ascending limb. This augmented $\mathrm{NaCl}$ delivery triggers a classical feedback mechanism that ultimately constricts the afferent arteriole and reduces GFR. Komlosi and colleagues discuss new insights into role of ATP and adenosine as paracrine mediators between macula densa cells and the afferent arteriole.

The second Emerging Topic-by Fleming, Kohlstedt, and Busse-examines the possibility that angiotensin converting enzyme (ACE), in addition to its classical vasoconstrictor role, may modulate intracellular signaling pathways. The mechanism may involve the ability of the cytoplasmic carboxy terminus of ACE to anchor several protein kinases. The authors also draw attention to the emerging role of ACE2, the carboxypeptidase activity of which leads to the formation of angiotensin metabolites that lead to vasodilation in several vascular beds, including that of the kidney.

The first two regular review articles in this issue deal with the glomerulus. The first-by Tryggvason and Wartiovaarafocuses on the role of the podocyte slit diaphragm in excluding proteins from the filtrate. Whereas previous work had stressed the role of the glomerular basement membrane in filtration, the present authors discuss the functional role of several newly identified proteins in the slit diaphragm (nephrin, neph-1, FAT, p-cadherin) and their role in conferring size and charge specificity to the glomerular barri- er. The absence of nephrin, for example, causes the massive protein loss associated with congenital nephrotic syndrome.

The second article that deals with glomerular function is by Ma, Pluznick and Sansom. These authors provide a timely update on our knowledge of ion channels in glomerular mesangial cells (MCs), the smooth-muscle-like cells in the core of the glomerulus. In health, the contraction of MCs can modulate the dynamics of glomerular filtration. In disease, MC proliferation plays a central role in the development of glomerulosclerosis In addition, the MCs respond to a wide variety of hormones and vasoactive agents. The wide variety of ion channels in MCs is likely to play important roles in all of these processes. Most of the channel information is based on work using patch-clamp studies of cultured MCs. Clarifying the physiological roles of these channels will require extending the studies to in vivo conditions.

Moving along the nephron, the article by Palacin and colleagues reviews recen developments in the physiology and pathophysiology of the heteromeric amino acid transporters (HATs). These proteins are unusual in that they are made up of two subunits, a "heavy" subunit from the SLC3 family and a "light" subuni from the $S L C 7$ family of transport proteins. Genetic defects in these transporters can give rise to defects in the reabsorption of amino acids in the proxima tubule, as well as the absorption of amino acids in the small intestine. Examples include cystinuria and Hartnup disease.

Still in the proximal tubule, the review by Hediger, Johnson, Miyazaki, and Endou examines the molecular mechanisms of urate transport. Deranged urate metabolism can lead to gout and uric acid kidney stones. The interpretation of earlier work had been difficult both because of the intrinsic complexity of the transport events (e.g., simultaneous secretion and absorption) and marked species differ- ences. Some clarification has now been provided by the identification of three transporters-URAT1, OAT1, and OAT3that are members of the SLC22 family. The fourth, MRP4, is a member of the ABC transporter family that also transports cAMP and cGMP.

The final two reviews in this issue deal with the control of electrolyte transport in the distal nephron. The first-by McCormick, Bhalla, Pao, and Pearceconsiders the role of serum- and glucocorticoid-regulated kinase 1 (SGK1) in mediating the action of aldosterone in stimulating transepithelial $\mathrm{Na}^{+}$transport. Evidence from heterologous expression studies in cultured cells, as well as $s g k 1$ knockout mice, suggests that aldosterone stimulates the synthesis of $s g k 1$, which in turn phosphorylates the Nedd4-2 ubiquitin ligase. The phosphorylation interferes with the ability of the ligase to interact with the apical epithelial $\mathrm{Na}^{+}$channel (ENaC), leading to the accumulation of the channel in the apical membrane. Thus, when challenged by $\mathrm{Na}^{+}$restriction, the $s g k 1$-knockout mouse is less able to conserve $\mathrm{Na}^{+}$.

In contrast to the previous review, which considers how $\mathrm{Na}^{+}$depletion leads to a rise in $\mathrm{Na}^{+}$reabsorption, the final the article in this issue-by Lim, Sterling, and Wang-investigates how dietary $\mathrm{K}^{+}$ restriction inhibits the secretion of $\mathrm{K}^{+}$. Recent evidence suggests that principal cells sense diminished $\mathrm{K}^{+}$intake and translate this signal into a stimulation of the Src protein tyrosine kinase. The subsequent phosphorylation of the main secretory $\mathrm{K}^{+}$channel (ROMK) enhances the internalization of ROMK from the apical membrane, thereby reducing $\mathrm{K}^{+}$secretion.

Although the subjects addressed in this issue of Physiology are of special importance for renal physiologists, each article has relevance for areas beyond the kidney. Whether you are a specialist looking for a critical review of the latest advances or a generalist taking a quick glance at what is new in renal physiology, we hope that you enjoy our April issue. 